\title{
LETTER DIBR-Synthesized Image Quality Assessment via Statistics of Edge Intensity and Orientation
}

\author{
Yu ZHOU ${ }^{\dagger}$, Leida LI ${ }^{\dagger a)}$, Ke GU ${ }^{\dagger \dagger}$, Zhaolin LU ${ }^{\dagger}$, Beijing CHEN ${ }^{\dagger \dagger}$, Nonmembers, and Lu TANG ${ }^{\dagger}$, Member
}

\begin{abstract}
SUMMARY Depth-image-based-rendering (DIBR) is a popular technique for view synthesis. The rendering process mainly introduces artifacts around edges, which leads to degraded quality. This letter proposes a DIBR-synthesized image quality metric by measuring the Statistics of both Edge Intensity and Orientation (SEIO). The Canny operator is first used to detect edges. Then the gradient maps are calculated, based on which the intensity and orientation of the edge pixels are computed for both the reference and synthesized images. The distance between the two intensity histograms and that between the two orientation histograms are computed. Finally, the two distances are pooled to obtain the overall quality score. Experimental results demonstrate the advantages of the presented method. key words: quality evaluation, DIBR, edge intensity, edge orientation
\end{abstract}

\section{Introduction}

The free-viewpoint television (FTV) has been arousing increasingly more interests [1]. This has boosted the development of view synthesis, which is employed to generate new views from existing views [2]. Depth-image-basedrendering (DIBR) is the most commonly used technique in view synthesis [3], which has great impact on the quality of synthesized views. Therefore, it is highly desirable to assess the quality of DIBR-synthesized images.

So far, only a few works have been done for the quality evaluation of DIBR-synthesized images. Bosc et al. [2] first calculated the distance map between reference and synthesized views. Then a threshold was used to obtain critical distorted regions, the mean structural similarity of which was defined as the quality score. In [4], the distortion map was first obtained by traditional 2D metrics. Then the texture, gradient and contrast maps were calculated as weighting maps of the distortion map. The weighted distortion maps were pooled to generate the quality score. In [5], the Morphological Wavelet Peak Signal-to-Noise Ratio (MW-PSNR) method was proposed. The multi-scale Mean Squared Error (MSE) was calculated based on the morphological wavelet decomposition. The MSEs on all

Manuscript received December 31, 2016.

Manuscript revised April 12, 2017.

Manuscript publicized May 1, 2017.

${ }^{\dagger}$ The authors are with School of Information and Control Engineering, China University of Mining and Technology, Xuzhou 221116, China.

${ }^{\dagger}$ The author is with the BJUT Faculty of Information Technology, Beijing University of Technology, Beijing 100124, China.

${ }^{\dagger \dagger}$ The author is with the School of Computer and Software, Nanjing University of Information Science and Technology, Nanjing 210044, China.

a)E-mail: lileida@cumt.edu.cn (Corresponding author)

DOI: 10.1587/transinf.2016EDL8255 scales were pooled to produce the score. The reduced version of MW-PSNR, namely RMW-PSNR [5] was presented, which was obtained by only using MSEs on higher scales. In [6], MW-PSNR was improved by using morphological pyramids, producing the Morphological Pyramid Peak Signalto-Noise Ratio (MP-PSNR) method. The reduced version of MP-PSNR (RMP-PSNR) [7] was also presented, which only used MSEs on higher scales. In [8], the blocks in reference and synthesized images were first registered. Then Kolmogorov-Smirnov distance between the matched blocks in wavelet domain was computed to obtain the quality score.

DIBR consists of warping and rendering. The warping step is to transform the reference view to the target view. In this stage, disoccluded regions are very likely to occur, mostly around edges, which are the areas invisible in reference views but emerge in synthesized views. The rendering step is to fill these regions using hole-filling techniques, the imperfection of which causes unnaturalness to edges. It is the most noticeable distortions in synthesized images. Most of the existing metrics are not effective in handling this kind of distortion. Hence, more effective metrics for DIBR synthesized images are needed.

Considering that DIBR mainly causes unnatural edges, this letter proposes a metric for DIBR-synthesized images based on statistics of edge intensity and orientation. Canny operator is first employed to locate edges. Then gradient maps are calculated. The intensity and orientation of edges in the gradient maps are computed. The edge intensity and orientation histograms of the reference and synthesized images are calculated and the distances between two intensity histograms and two orientation histograms are computed. Finally, two distances are pooled to generate the quality score. Experiment results verify the effectiveness of the presented metric.

\section{Proposed Metric}

In this work, the edge intensity and orientation histograms are generated using the gradients in the edge regions. In this paper, the edge of an input image $\mathbf{F}(M \times N)$ is first detected using the Canny operator [9]. The binary edge map is denoted by $\mathbf{E}$.

The edge intensity map is calculated as:

$$
\mathbf{G}=\frac{\left|\mathbf{G}_{x}+\mathbf{G}_{y}\right|}{2},
$$

where $\mathbf{G}_{x}$ and $\mathbf{G}_{y}$ are the horizontal and vertical gradients, 
respectively, which are computed as:

$$
\mathbf{G}_{x}=\mathbf{H} * \mathbf{F}, \mathbf{G}_{y}=\mathbf{H}^{T} * \mathbf{F},
$$

where $*$ and $\mathrm{T}$ denote the convolution and transpose, respectively, $\mathbf{H}$ is the gradient operator:

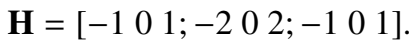

As aforementioned, only the gradients in the edge regions detected by the Canny operator are used. Therefore, the edge intensity map is defined as:

$$
\mathbf{I}=\mathbf{G} \times \mathbf{E},
$$

where $\times$ denotes the pointwise multiplication.

The orientation of an edge pixel $(x, y)$ is defined as:

$$
\mathbf{O}(x, y)=\arctan \left(\frac{\mathbf{G}_{y}(x, y)}{\mathbf{G}_{x}(x, y)+c}\right) \frac{180^{\circ}}{\pi},
$$

where $c$ is a small constant for numerical stability.

\subsection{Statistics of Edge Intensity}

The range of edge intensity is $[0,255]$. We divide it into $p$ equally spanned bins. All pixels in each bin are used to generate the intensity histogram. Suppose the intensity histograms of the reference and synthesized images are $\mathbf{U}_{r}$ and $\mathbf{U}_{s}$, respectively. The average distance between them is defined as the edge intensity score:

$$
Q_{I}=\frac{\sum_{i=1}^{p}\left|\mathbf{U}_{s}(i)-\mathbf{U}_{r}(i)\right|}{n},
$$

where $i$ denotes the index of a bin and $n$ is the total number of edge pixels.

\subsection{Statistics of Edge Orientation}

The range of edge orientation is $\left[-180^{\circ}, 180^{\circ}\right]$. We divide it into $q$ equally spanned bins. All pixels in each bin are employed to generate the orientation histogram. Suppose the orientation histograms of the reference and synthesized images are $\mathbf{H}_{r}$ and $\mathbf{H}_{s}$, respectively. Then the average distance between $\mathbf{H}_{r}$ and $\mathbf{H}_{s}$ is defined as the edge orientation score:

$$
Q_{O}=\frac{\sum_{i=1}^{q}\left|\mathbf{H}_{s}(i)-\mathbf{H}_{r}(i)\right|}{n} .
$$

Figure 1 shows a reference image and its synthesized image, together with their histograms of edge intensity and orientation. It is observed that the edge intensity and orientation histograms of the synthesized image are different from those of the reference image. This demonstrates that the imperfect rendering process does affect the statistics of edge intensity and orientation.

\subsection{Pooling}

The statistics of edge intensity and orientation are combined
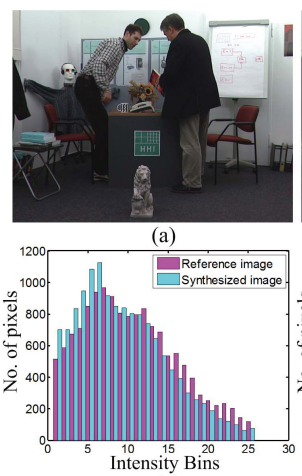

(c)

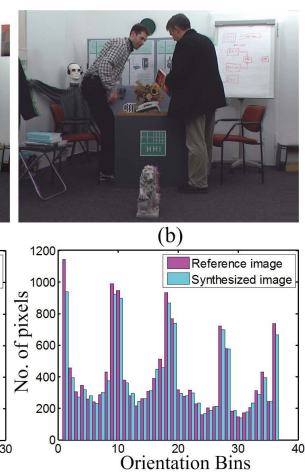

(d)
Fig. 1 Edge intensity and orientation histograms of reference and synthesized images. (a) Reference image; (b) synthesized image; (c)-(d) edge intensity and orientation histograms of (a) and (b).

to define the final quality score:

$$
Q=\alpha Q_{I}+\beta Q_{O},
$$

where $\alpha$ and $\beta$ are parameters to balance the relative importance of the two components. In this work, we experientially set $\alpha$ and $\beta$ to 0.65 and 0.35 . The reason $\alpha$ is bigger than $\beta$ is that the edge intensity plays a more important role than edge orientation in evaluating the quality of synthesized images. This can be seen from Fig. 1, where the distance between two edge intensity histograms is more significant than the distance between two edge orientation histograms.

\section{Experimental Results}

\subsection{Experiment Settings}

The IRCCyN/IVC DIBR image database [2] is used to evaluate the performance of the proposed method. The database consists of 12 reference, 84 synthesized images and the subjective scores, namely Mean Opinion Scores (MOS). The MOS values are obtained by the Absolute Category RatingHidden Reference (ACR-HR) method [10], which is a noreference method. Therefore, in order to ensure the fair evaluation of full-reference methods, the Difference Mean Opinion Scores (DMOS) are calculated using the MOS values of both the reference and synthesized images [11].

For performance evaluation, we use four popular criteria, which are Pearson Linear Correlation Coefficient (PLCC), Root Mean Square Error (RMSE), Kendalls Rank Correlation Coefficient (KRCC) and Spearman Rank order Correlation Coefficient (SRCC). Among them, PLCC and RMSE are employed to measure the prediction accuracy, while the latter two are used to measure the prediction monotonicity. Higher values of PLCC, SRCC, KRCC and a lower value of RMSE indicate a better performance. They are calculated after the five-parameter logistic mapping [12]:

$$
f(x)=\tau_{1}\left(\frac{1}{2}-\frac{1}{1+e^{\tau_{2}\left(x-\tau_{3}\right)}}\right)+\tau_{4} x+\tau_{5},
$$

where $x$ and $f(x)$ denote the predicted and subjective scores, 

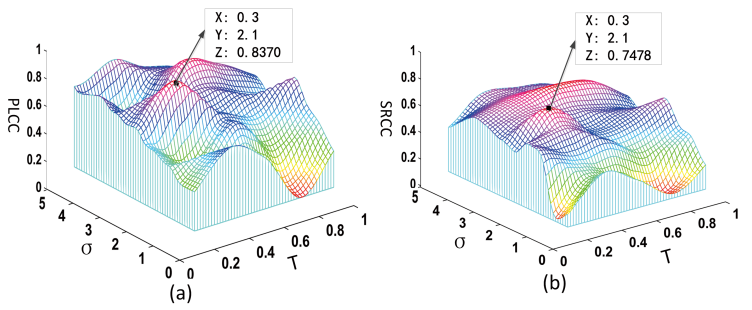

Fig. 2 PLCC and SRCC values using different parameters $\sigma$ and T.
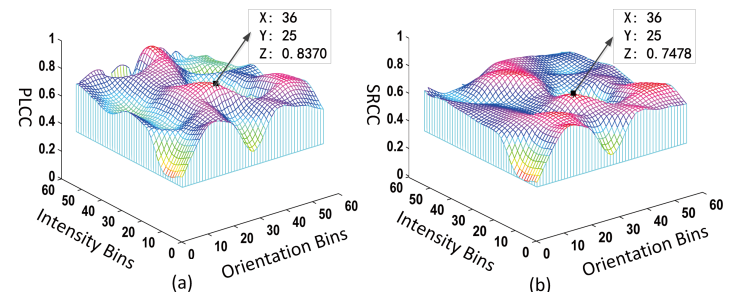

Fig.3 PLCC and SRCC values using different bin numbers.

and $\tau_{i}, i=1,2,3,4,5$, are the parameters to be fitted.

\subsection{Parameters Settings}

\subsubsection{Impact of Parameters in Canny Operator}

During the edge detection using Canny operator, two parameters need to be determined, namely the standard deviation $\sigma$ of the Gaussian filter and the threshold T, which are set empirically in implementation. Figure 2 shows the PLCC and SRCC values obtained using different combinations of $\sigma$ and T. The coordinates of their peak values are marked in the figures.

From Fig. 2, we can see that PLCC and SRCC values both reach their peaks at $\sigma=2.1$ and $\mathrm{T}=0.3$, so all experiments are based on these values.

\subsubsection{Impact of Histogram Bin Numbers}

Figure 3 shows the performances of the method with different bin numbers of the orientation and intensity histograms.

It can be seen from Fig. 3 that the number of bins has effects on the metric performance and when the bin numbers of the orientation and intensity histograms are 36 and 25 , respectively, the metric achieves the best performance. Therefore, 36 and 25 are deemed as the best number of bins of the orientation and intensity histograms.

\subsection{Performance Evaluation}

To demonstrate the superiority of the presented metric, we compare it with traditional 2D and existing DIBR quality metrics. Traditional approaches include PSNR, SSIM [13], VSI [14], IGM [15], ADD-SSIM [16], LTG [17], and QASD [18], while DIBR quality methods are Bosc [2], VSQA [4], MW-PSNR [5], RMW-PSNR [5], MP-PSNR [6],
Table 1 Performances of traditional 2D, existing DIBR quality metrics and the proposed method.

\begin{tabular}{cccccc}
\hline Metric & Type & PLCC & SRCC & KRCC & RMSE \\
\hline PNSR & 2D & 0.5098 & 0.4628 & 0.3321 & 0.5728 \\
SSIM [13] & 2D & 0.5644 & 0.5245 & 0.3650 & 0.5496 \\
VSI [14] & 2D & 0.5218 & 0.3801 & 0.2588 & 0.5680 \\
IGM [15] & 2D & 0.5476 & 0.4893 & 0.3385 & 0.5571 \\
ADD-SSIM [16] & 2D & 0.6470 & 0.5611 & 0.4141 & 0.5077 \\
LTG [17] & 2D & 0.5311 & 0.4139 & 0.2900 & 0.5642 \\
QASD [18] & 2D & 0.5453 & 0.5098 & 0.3691 & 0.5581 \\
\hline Bosc [2] & DIBR & 0.5843 & 0.4905 & 0.3414 & 0.5403 \\
VSQA [4] & DIBR & 0.5742 & 0.5233 & 0.3673 & 0.5451 \\
MW-PSNR [5] & DIBR & 0.5622 & 0.5757 & 0.4378 & 0.5506 \\
RMW-PSNR [5] & DIBR & 0.5744 & 0.6245 & 0.4960 & 0.5450 \\
MP-PSNR [6] & DIBR & 0.6174 & 0.6227 & 0.4833 & 0.5238 \\
RMP-PSNR [7] & DIBR & 0.6772 & 0.6634 & 0.5382 & 0.4899 \\
3DSwIM [8] & DIBR & 0.6584 & 0.6156 & 0.4496 & 0.5011 \\
Proposed SEIO & DIBR & $\mathbf{0 . 8 3 7 0}$ & $\mathbf{0 . 7 4 7 8}$ & $\mathbf{0 . 5 5 3 2}$ & $\mathbf{0 . 3 6 4 2}$ \\
\hline & & & & &
\end{tabular}
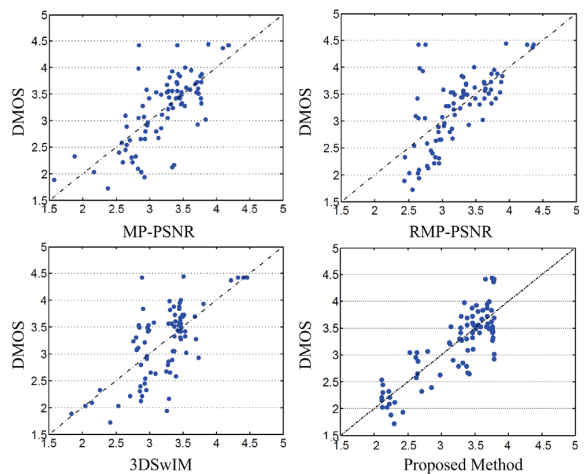

Fig. 4 Scatter plots of the subjective scores versus converted objective estimations of the top four DIBR quality metrics in Table 1.

RMP-PSNR [7] and 3DSwIM [8]. Table 1 summarizes the experiment results, where the best results are marked in boldface.

Table 1 shows that the proposed metric performs better than both traditional and existing DIBR quality methods. Existing DIBR quality methods perform better than traditional metrics. However, they are still not ideal. By contrast, the presented SEIO achieves much better performance.

For intuition, Fig. 4 shows the scatter plots between subjective and objective scores obtained from the top four DIBR quality methods. For a better metric, the points are supposed to distribute more closely to the fitting curve [19]. It is observed that the points generated from the proposed SEIO spread more intensively around the curve. This manifests that the scores predicted by the presented method have better consistency with subjective scores.

\subsection{Component Evaluation}

The presented method consists of statistic modules of edge intensity (SEI) and orientation (SEO). Therefore, it is necessary to testify the necessity of the combination of two components. To this end, we test the performances of the methods separately using SEI, SEO and together SEIO. The performances are shown in Fig. 5. 


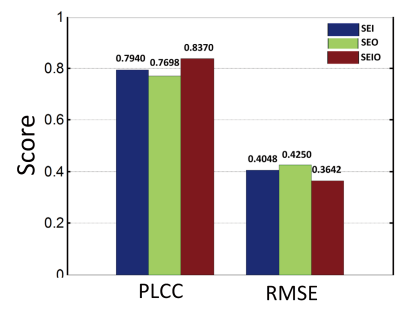

Fig.5 Performances of the proposed method using SEI module and SEO module separately, and combining two modules.

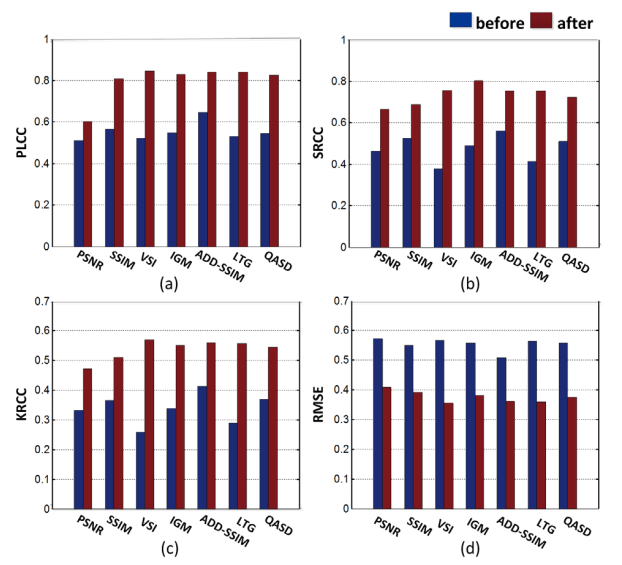

Fig. 6 Performances of traditional 2D quality metrics before and after integrating the SEIO module.

It can be seen from the figure that the metric integrating both components achieves the best performance. This indicates the necessity and rationality of the combination of two components. Moreover, the method only using SEI performs better than that only using SEO, which indicates that SEI contributions more than the SEO. This is also the reason why $\alpha$ is bigger than $\beta$ in Eq. (8).

\subsection{Application}

Traditional 2D metrics are not designed for view synthesis, so they have limited performances. The proposed method can be integrated into these metrics by multiplication operation to improve their performances. Figure 6 shows the performances of traditional metrics before and after integrating the proposed metric. It is observed that after integrating SEIO, the performances of all traditional methods have significant improvements. This further demonstrates the effectiveness of the proposed method.

\section{Conclusion}

In this letter, we have proposed a DIBR-synthesized image quality metric based on the statistics of edge intensity and orientation. It is based on the observation that the imperfect rendering in DIBR causes unnaturalness of edges. Statistics of edge intensity and orientation have been proposed to measure the edge unnaturalness. Experiment results have demonstrated the effectiveness of the proposed method. Moreover, the presented method has been utilized to improve the performances of traditional metrics, and very promising results have been obtained.

\section{Acknowledgements}

This work is supported by the Fundamental Research Funds for the Central Universities under Grant 2017XKQY084.

\section{References}

[1] Y.-C. Fan, P.-K. Huang, and D.-W. Shen, "3DTV depth map reconstruction based on structured light scheme," IEEE Int. Instrum. Meas. Technol. Conf. (I2MTC), pp.835-838, 2013.

[2] E. Bosc, R. Pépion, P.L. Callet, M. Koppel, P. Ndjiki-Nya, M. Pressigout, and L. Morin, "Towards a new quality metric for 3-D synthesized view assessment," IEEE J. Sel. Topics Signal Process., vol.5, no.7, pp.1332-1343, 2011.

[3] Y.J. Jung, H.G. Kim, and Y.M. Ro, "Critical binocular asymmetry measure for the perceptual quality assessment of synthesized stereo 3D images in view synthesis," IEEE Trans. Circuits Syst. Video Technol., vol.26, no.7, pp.1201-1214, 2016.

[4] P.H. Conze, P. Robert, and L. Morin, "Objective view synthesis quality assessment," Proc. Electron. Imag. Int. Society for Optics and Photonics, pp.8288-8256, 2012.

[5] D. Sandic-Stankovic, D. Kukolj, and P.L. Callet, "DIBR synthesized image quality assessment based on morphological wavelets," IEEE Int. Workshop on Quality of Multimedia Experience, pp.1-6, 2015.

[6] D. Sandic-Stankovic, D. Kukolj, and P.L. Callet, "DIBR synthesized image quality assessment based on morphological pyramids," The True Vision - Capture, Transmission and Display of 3D Video, pp.1-4, 2015.

[7] D. Sandić-Stanković, D. Kukolj, and P.L. Callet, "Multi-scale synthesized view assessment based on morphological pyramids," J. Electr. Eng., vol.67, no.1, pp.3-11, 2016.

[8] F. Battisti, E. Bosc, M. Carli, P.L. Callet, and S. Perugia, "Objective image quality assessment of 3D synthesized views," Sig. Process.: Image Commun., vol.30, pp.78-88, 2015.

[9] J. Canny, "A computational approach to edge detection." IEEE Trans. Pattern Anal. Mach. Intell., vol.PAMI-8. no.6, pp.679-698, 1986.

[10] ITU-T p.910, "Subjective video quality assessment methods for multimedia applications," 1997.

[11] G. Cermak, L. Thorpe, and M. Pinson, Test plan for evaluation of video quality models for use with high definition TV content, Video Quality Experts Group, 2009.

[12] X. Guo, L. Huang, K. Gu, L. Li, Z. Zhou, and L. Tang, " Naturalization of screen content images for enhanced quality evaluation," IEICE Trans. Inf. \& Syst., vol.E100-D, no.3, pp.574-577, March 2016.

[13] Z. Wang, A.C. Bovik, H.R. Sheikh, and E.P. Simoncelli, "Image quality assessment: From error visibility to structural similarity," IEEE Trans. Image Process., vol.13, no.4, pp.600-612, 2004.

[14] L. Zhang, Y. Shen, and H. Li, "VSI: A visual saliency-induced index for perceptual image quality assessment," IEEE Trans. Image Process., vol.23, no.10, pp.4270-4280, 2014.

[15] J. Wu, W. Lin, G. Shi, and A. Liu, "Perceptual quality metric with internal generative mechanism," IEEE Trans. Image Process., vol.22, no.1, pp.43-54, 2013.

[16] K. Gu, S. Wang, G. Zhai, W. Lin, X. Yang, and W. Zhang, "Analysis of distortion distribution for pooling in image quality prediction," IEEE Trans. Broadcast., vol.62, no.2, pp.446-456, 2016.

[17] K. Gu, G. Zhai, X. Yang, and W. Zhang, "An efficient color image quality metric with local-tuned-global model," Proc. IEEE Int. Conf. Image Process., pp.506-510, 2014. 
[18] L. Li, H. Cao, Y. Zhang, W. Lin, A.C. Kot, and X. Sun, "Sparse representation based image quality index with adaptive subdictionaries," IEEE Trans. Image Process., vol.25, no.8, pp.37753786, 2016.
[19] L. Li, Y. Zhou, W. Lin, J. Wu, X. Zhang, and B. Chen, "No-reference quality assessment of deblocked images," Neurocomputing, vol.177, pp.572-584, 2016. 